complaints should be based on regular PPE focussing on the musculoskeletal system as well as internal medical and cardiovascular screening.

\section{STRESS FRACTURES DURING TOP-LEVEL INTERNATIONAL ATHLETICS CHAMPIONSHIPS}

${ }^{1,2,3}$ Pascal Edouard, ${ }^{4,5}$ Anders Vinther. ${ }^{1}$ Inter-university Laboratory of Human Movement Science (LIBM EA 7424), University of Lyon, University Jean Monnet, Saint-Etienne, France; ${ }^{2}$ Department of Clinical and Exercise Physiology, Sports Medicine Unit, University Hospital of Saint-Etienne, Faculty of Medicine, Saint-Etienne, France; ${ }^{3}$ European Athletics Medical and Anti Doping Commission, European Athletics Association (EAA), Lausanne, Switzerland; ${ }^{4}$ Department of Physiotherapy and Occupational Therapy, Copenhagen University Hospital, Herlev and Gentofte, Copenhagen, Denmark; ${ }^{5}$ QD-Research unit, Copenhagen University Hospital, Herlev and Gentofte, Copenhagen, Denmark

\subsection{6/bjsports-2021-IOC.241}

Background Stress fracture is a frequent injury among athletics athletes. During international Athletics championships, although stress fractures represented a small percentage of all injuries $(2.9 \%$ of all injuries and $4.9 \%$ of in-competition timeloss injuries for female athletes), it exists and should not be neglected, because it could be one symptom of Relative Energy Deficiency in Sport.

Objective To specifically analyse stress fractures during top-level international Athletics championships from 2007 to 2019.

Design Prospective study.

Setting 21 international championships from 2007 to 2019.

Participants 26281 (14130 male and 12151 female) international-level registered athletes.

Main Outcome Measurements The national medical team and the local organizing committee physicians reported all injuries daily on a standardised injury report form during 21 international championships. Only stress fractures were included in the descriptive analysis.

Results During the 21 international athletics championships, a total of 36 stress fractures were reported, representing 1.6\% of all reported injuries. 14 were in male and 22 in female athletes, and $54 \%$ in endurance and $46 \%$ in explosive disciplines. The overall stress fracture incidence was 1.4 per 1000 registered athletes $(95 \% \mathrm{CI}=1.0-1.8)$. The relative risk was almost doubled in female compared to male athletes although this was not statistically significant (relative risk $(R R)=1.83$, $95 \% \mathrm{CI}=0.94-3.57)$. Most of stress fractures involved the lower extremity (92\%). In female athletes, $46 \%$ were located at the lower leg and $41 \%$ at the foot, compared to $14 \%$ and $64 \%$, respectively for male athletes. More than half of the stress fractures were classified as severe injuries (i.e. estimated number of days of absence $>28$ days).

Conclusions Stress fractures also occurred during major international athletics championships, representing a severe injury, with sex differences in location and most likely also in overall risk.

\section{EXPERT OPINION ON THE ASSESSMENT AND MANAGEMENT OF CALF MUSCLE STRAIN INJURIES IN SPORT}

Brady Green, Anthony Schache, Jodie McClelland, Adam Semciw, Tania Pizzari. La Trobe Sport and Exercise Medicine Research Centre, Melbourne, Australia

$10.1136 /$ bjsports-2021-IOC. 242
Background Despite being a common cause of time loss, information regarding best practice for calf muscle strain injuries (CMSI) in sport is scarce.

Objective To establish best practice for the assessment and management of CMSI.

Design Qualitative.

Setting In-depth interviews.

Patients (or Participants) 20 expert medical professionals working in elite sport and/or researchers specialising in the field; representing seven countries and seven sports.

Interventions (or Assessment of Risk Factors) Semi-structured interviews using a schedule of questions canvassing pre-identified topics. Thematic coding to analyse findings.

Main Outcome Measurements Data were evaluated in three key areas: (i) injury characteristics, (ii) injury management, and (iii) injury prevention.

Results CMSI have unique injury characteristics compared to other common muscle strain injuries (e.g. hamstring), but a criteria-based approach can assist forming the most accurate impression of prognosis. Similarly, a structured approach should be followed to ensure the athlete returns to a high level of performance and the risk of re-injury is minimized, focusing on: re-strengthening, plyometric and ballistic exercises, as well as running-based reconditioning specific to the sport. For the best chance to prevent index CMSI, strategies should span multiple domains of athlete management: screening and monitoring, field-based exposure (e.g. workload data), and off-field interventions (e.g. strengthening). Injury prevention strategies should be tailored to the individual, considering extrinsic (the sport, position played, club culture/coach expectations) and intrinsic (previous injury history, age, training history) factors that may increase susceptibility to CMSI.

Conclusions Knowledge about the unique injury characteristics of CSMI can clarify the likely prognosis and best approach to rehabilitation. Practitioners attempting to prevent CMSI should use a multi-faceted approach given that the aetiology of CMSI is complex and often unique to the individual.

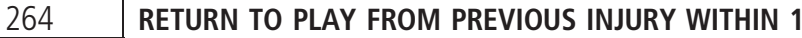 YEARS MAY BE AN IMPORTANT RISK FACTORS TO BE CONSIDERED IN ORDER TO PREVENT RECURRENT INJURY DURING MAJOR EVENTS FOR YOUNG ATHLETES}

\footnotetext{
${ }^{4,5}$ Youngjun Kim, ${ }^{4,5}$ Sejun Kim, ${ }^{1,2}$ Hee Seong Jeong, ${ }^{1,2,3}$ Sae Yong Lee. ${ }^{1}$ Yonsei Institute of Sports Science and Exercise Medicine, Yonsei University, Seoul, South Korea (Republic of); ${ }^{2}$ Department of Physical Education, Yonsei University, Seoul, South Korea (Republic of); ${ }^{3}$ Institute of Convergence Science, Yonsei University, Seoul, South Korea (Republic of); ${ }^{4}$ Medical and Science department, Korean Sports and Olympic Committee, Jincheon, South Korea (Republic of); ${ }^{5}$ Medical center, Jincheon National Training Center, Jincheon, South Korea (Republic of)
}

\subsection{6/bjsports-2021-IOC.243}

Background Investigating the relationship between time from return to play (RTP) and injury characteristics may provide an important information to preventing a recurrent injury in mass sports event.

Objective To investigate rate of recurrent injuries, and injury types and parts of recurrent injuries during 2017 Summer and Winter Universiade Games.

Design Prospective epidemiological study.

Setting Injuries were reported during 2017 Summer (2017S) and Winter Universiade (2017W) Games by medical staffs. 
Main Outcome Measurements Using typical IOC injury report data and data extracted from Team Korea EMR(electronic medical records) system tracking injuries of all national team, epidemiological datum were analyzed. These include injury body parts, types, RTP within 1 year and RTP over 1 year. Results A total of 80 [(47 recurrent (59\%)] and 39 [17 recurrent (44\%)] injuries were identified during $2017 \mathrm{~S}$ and $2017 \mathrm{~W}$ repectively. Top two injured parts in $2017 \mathrm{~S}$ were lumbar $(n=9,11 \%)$ and ankle $(n=8,10 \%)$ in within 1 year from RTP whereas shoulder $(n=5,6 \%)$ and lumbar $(n=4,5 \%)$ in over 1years from RTP. Top two injured types in $2017 \mathrm{~S}$ were strain $(n=11,14 \%)$ and tendonitis $(n=9,11 \%)$ in within 1 year from RTP whereas strain $(n=6,8 \%)$ and nerve injury $(n=3,4 \%)$ in over 1years of RTP. Top two injured parts in $2017 \mathrm{~W}$ were knee $(n=5,16 \%)$ and lumbar $(n=3,9 \%)$ in within 1 year from RTP whereas lumbar $(n=4,13 \%)$ and hip $(n=1,3 \%)$ in over 1years of RTP. Top two injured types in $2017 \mathrm{~W}$ were sprain $(n=7,22 \%)$, meniscus $(n=2,6 \%)$ in within 1 year RTP whereas sprain $(n=5,16 \%)$ and nerve injury $(n=1,3 \%)$ in over 1years from RTP.

Conclusions Athletes who RTP within a year should be cautious to be re-injured during major sports events. Major body parts to be considered are lumbar, knee, and ankle with muscle and ligament injuries.

\section{COMMUNITY-BASED REHABILITATION IMPLEMENTATION FRAMEWORK TO ADDRESS INJURIES \& ITS RISKS AMONG RUNNERS IN UNDER-RESOURCED COMMUNITIES: DELPHI CONSENSUS}

Siyabonga Henry Kunene. University of the Witwatersrand, Johannesburg, South Africa

10.1136/bjsports-2021-IOC.244

Background Athletes in low socioeconomic communities generally present with poor health outcomes as compared to those in privileged communities. A high prevalence and risks of sports-related injuries have been reported among runners in under-resourced communities. These have a negative impact on their quality of life and level of sports participation.

Objective To develop a community-based rehabilitation implementation framework for sports-related injuries among runners in under-resourced communities, using South African communities as an example.

Design The Delphi technique was used in building consensus on the appropriate framework.

Setting The study was based in South Africa.

Participants Sport medicine experts (including sports physicians, physiotherapists, biokiniticists, sports therapists, psychologists, nutritionists/dieticians, podiatrists) in treating and rehabilitating sports-related injuries were recruited to participate.

Main Outcome Measurements The questionnaire was then sent to participants to make comments and rate each item using a four-point Likert scale.

Results 19 experts participated in the study: 3 physicians, 5 physiotherapists, 2 sports therapists, 2 biokineticists, 2 podiatrists, 2 dieticians, a d 2 psychologists. 10 were females and 9 were males of which 13 were aged $36-55$ years. All experts were based in South African with 11 - 20 years of clinical experience. The Delphi process yielded four core framework items. These were: 1) the establishment of transdisciplinary rehabilitation teams, 2) upskilling of available clinicians, their assistants, and trainers, 3) implementation of a communitybased rehabiliation programme at low-level/no-cost and 4) referral of cases to secondary/tertiary institution for further intervention.

Conclusions Consensus was reached for a comprehensive rehabilitation framework aimed at addressing the specific needs of athletes in under-resourced communities where rehabilitation services are scarce. A further study to test the feasibility of the agreed-upon framework is underway.

\section{SLOW, UNILATERAL ELASTIC RESISTANCE TRAINING ELICITS A CROSS-EDUCATION EFFECTS IN THE CONTRALATERAL LIMB, MEASURED BY DYNAMOMETRY AND ELECTROMYOGRAPHY}

\footnotetext{
${ }^{1,2}$ Stephen Loke, 1,2 Bhavesh Kumar, 1,2Kushal Varma, 1,2 Krishan Patel, 1,2 Henry Beckett,

${ }^{1,2}$ Flaminia Ronca. 'Institute of Sport, Exercise and Health (ISEH), London, UK; ${ }^{2}$ University College London (UCL), London, UK
}

\subsection{6/bjsports-2021-IOC.245}

Background Cross-education occurs when unilateral resistance training results in a strength transfer to the contralateral limb. This study explores the application of cross-education in injury rehabilitation.

Objective This study investigated the effectiveness of unilateral elastic resistance training on shoulder and elbow strength and neuromuscular activation in the contralateral limb. The secondary aim was to identify the impact of training velocity on these adaptations.

Design A within-subject randomised controlled trial.

Setting University students were tested in the lab before and after a home-based training programme.

Patients (or Participants) Twenty-nine healthy, sedentary, young adults (21.2 \pm 0.9 years) were match randomised into four intervention groups according to training arm dominance and movement velocity $(60 \%$ vs. $240 \%$ s): fast-dominant, fast-nondominant, slow-dominant and slow-nondominant.

Interventions (or Assessment of Risk Factors) Participants completed a home-based 6-week unilateral strength programme including resistance tubing exercises of shoulder internal/external rotation and elbow flexion/extension.

Main Outcome Measurements Peak torque (PT) and surface electromyography (EMG) was recorded during maximal voluntary isometric contractions (MVIC), isokinetic concentric $60 \%$ and $240 \%$ contractions two weeks before intervention, preintervention, and post-intervention.

Results All groups had significant PT increases in untrained arm elbow extension at $60 \% \mathrm{~s}$ and $240 \%$ s $(\mathrm{P}<0.01)$. The slow and the dominant arm groups experienced significant PT improvements $(\mathrm{P}<0.05)$ in internal rotation at $240 \%$ s.

The trained arm of the slow-dominant group had significant increases in EMG peak amplitude for supraspinatus external rotation $60 \%$ and triceps elbow extension MVIC $(\mathrm{P}<0.05)$, with the untrained arm also increasing significantly in elbow extension MVIC. The dominant groups showed significantly greater peak EMG increases than the non-dominant groups in trained supraspinatus $240 \% \mathrm{~s}$ and untrained teres major MVIC $(\mathrm{P}<0.05)$.

Conclusions Unilateral elastic resistance training produces strength and neuromuscular benefits in both arms, suggesting that home-based interventions are a potential rehabilitation adjunct in preventing reinjuries. Cross-education is greatest when the dominant arm is trained at slower velocities. 